\title{
ORT_11 - Naphthoquinone as P2X7 receptor inhibitors: A preliminary study to assess anti- inflammatory activity in vivo
}

\author{
Juliana Vieira Faria ${ }^{1 *}$; Robson Xavier Faria ${ }^{2}$. \\ ${ }^{1}$ UFF - Universidade Federal Fluminense; \\ ${ }^{2}$ Fiocruz/IOC.
}

Introduction: The $\mathrm{P} 2 \mathrm{X} 7$ purinergic receptor (P2X7R) is a channel physiologically activated by extracellular adenosine 5'-triphosphate (ATP) and distributed in mammals, including hematopoietic cells, such as macrophages and microglia. Brief exposure to ATP allows cations, such as $\mathrm{Ca} 2+, \mathrm{Na}+$, and $\mathrm{K}+$, to pass through the membrane. A prolonged stimulation induces a pore's formation allowing the entry of molecules of up to $900 \mathrm{Da}$. Besides, P2X7R is responsible for regulating other cellular events, including the release of pro-inflammatory cytokines (IL-1 $\beta$, IL-6, TNF- $\alpha$ ) and cell death. Once released, these cytokines mediate the generation and control of immune and inflammatory responses. The therapeutic control of inflammation by blocking the IL- $1 \beta$ release pathway by inhibiting P2X7R has been widely studied for the clinical treatment of inflammatory and autoimmune diseases, such as neuropathic pain and rheumatoid arthritis. However, current treatments are palliative and have several associated side effects. Pharmacologically, the P2X7 receptor has some antagonists. However, they have low efficacy in human clinical trials when compared to the standard drug. Therefore, it is necessary to discover new antagonists for this receptor.

Objective: In this context, we performed screenings using a series of 11 naphthoquinone derivatives, series 135 (135a, 135b, 135c, 135d, 135e, 135f, 135g, 135h, 135i, 135j, and 1351), to evaluate its activity at the $\mathrm{P} 2 \mathrm{X} 7$ receptor.

Methodology: We evaluated the P2X7 receptor inhibition using Ethidium Bromide (EB) uptake assay. The molecules were tested in an initial concentration at $10 \mu \mathrm{M}$. The tests were realized on three different days. We used the Graphpad Prism software (ANOVA test, followed by the Turkey post-test) for statistical analysis.

Results: Positive Control using Triton-X 100 promoted the maximal dye uptake (83.15 \pm 18.40$)$. No treated cell exhibited a basal dye entry $(21.22 \pm 11.28)$. ATP induced a prominent EB uptake $(77.35 \pm$ $16,34)$, and as expected, BBG inhibited this response $(16.80 \pm 6.951)$. The prototypes pretreated for 5 minutes before ATP application reduced the ATP response 135a $(54.70 \pm 23.44), 135 \mathrm{~b}(44.10 \pm 30.14)$, $135 \mathrm{c}(47.98 \pm 13.94), 135 \mathrm{~d}(32.04 \pm 6.157), 135 \mathrm{e}(43.2 \pm 12.39), 135 \mathrm{f}(48.69 \pm 21.24), 135 \mathrm{~g}(63.24$ $\pm 22.81), 135 \mathrm{~h}(49.65 \pm 16.10), 135 \mathrm{i}(53.65 \pm 17.48), 135 \mathrm{j}(51.08 \pm 17.64)$, and 1351 (27.40 \pm 23.60$)$. However, the molecules $135 \mathrm{~d}$ and 1351 inhibited with statistical significance.

Conclusion: We can conclude that the molecules $135 \mathrm{~d}$ and 1351 are the prototypes selected for further studies.

Keywords: Purinergic; ATP; P2X7 